\title{
FREQUENCY- AND TIME-DOMAIN EXPRESSIONS FOR TRANSFER FUNCTIONS AND IMPULSE RESPONSES RELATED TO THE WAVEGUIDE PROPAGATION
}

\author{
By \\ A.S. Omar and A.H. Kamel \\ Chair of Microwave and Communication Engineering, University of Magdeburg \\ P.O. Box 4120, D-39016 Magdeburg, Germany
}

\begin{abstract}
Analytic expressions for the frequency dependent transfer functions and their time dependent inverse Fourier transforms, which represent the forward transmission and the multiple reflection in a waveguide section are derived. Making use of these analytic expressions, the multiple reflection of a narrowband Gaussian pulse in a waveguide section with different load-side and generator-side terminations is graphically demonstrated. This shows that early reflections are in general less dispersed than later ones. For electrically long waveguide sections operating high enough above their cutoff frequency, the different reflections are temporally distinguishable. On the other hand, in both electrically short and evanescent waveguide sections, the temporal overlapping between the different reflections is so strong that their individual identification is not possible. It is also shown that recently reported superluminal propagation of pulse peaks can take place in electrically short evanescent waveguide sections only. Increasing the waveguide length beyond a certain limit destroys such a superluminality.
\end{abstract}

\section{INTRODUCTION}

The multiple reflection at mismatched ends of a transmission medium may lead to sever signal distortion even if the transmission medium is not dispersive. The reason for such a distortion is the interference between the signal and its echoes if the latter are strong and temporally overlapped. The strength of the signal echoes is determined by the degree of mismatching at both sides of the transmission channel. On the other hand, temporal overlapping between the signal and the individual echoes depends on their temporal extension compared to the channel transit time (channel length divided by propagation velocity). It is therefore important to consider these issues quantitatively in order to be able to determine the necessity of distortion compensation.

TE and TM transmissions in waveguides suffer generally from two types of distortions. The first is due to the nonlinear frequency dependence of the propagation constant, which is mainly a phase distortion. The second, which may accompany TEM transmission as well, is due to the mismatching at the load (receiver) side and/or the generator (transmitter) side. This type represents in fact both amplitude and phase distortions. Both types affect the transmission of information signals through waveguide channels and may lead to a drastic increase in the symbol-error rate in case of digital transmission, which consequently leads to a reduction in the effective data rate (refer to any standard textbook on digital communication and information theory, e.g., [1]).

Because of their temporal and spectral compactness, Gaussian pulses are sometimes preferred in digital communication. A digital symbol is represented by the amplitude, the frequency or 
the phase of an RF carrier signal which is modulated by a base-band Gaussian pulse (ASK, FSK or PSK, respectively). The GMSK (Gaussian Minimum Shift Keying) is an example for a binary transmission. The dispersive transmission of such symbols in waveguides may change one symbol into the other giving rise to symbol-error (bit-error in case of binary transmission).

Both transient and steady-state analysis of signal transmission in waveguides have been comprehensively presented in the open literature (e.g., [2]-[7]). Transient behavior of the multiple reflection in a waveguide section has been considered in our recent publication [6] only qualitatively. It is the goal of the present contribution to study this issue quantitatively and in more details. The contribution begins with a derivation of analytic expressions for the frequency dependent transfer functions and their time dependent inverse Fourier transforms, which represent the forward transmission and the multiple reflection in a waveguide section. The multiple reflection of a narrowband Gaussian pulse in a waveguide section with different load-side and generator-side terminations is then investigated. Case studies are then presented which graphically demonstrate the effect of the different distortion mechanisms on the pulse shape.

\section{BASIC FORMULATION}

Let us consider an empty waveguide section of length $L$ transmitting information signals over one of its TE modes from a source (transmitter) to a load (receiver). Restricting the analysis to TE modes doesn't however affect its generality, as the behavior of TM modes can be obtained by simply exchanging the roles of the electric and magnetic fields. Fig. 1 shows an equivalent transmission line, for which the line voltage and current are to be interpreted as merely the axial dependences of the waveguide transverse electric and magnetic field, respectively. The propagation constant $\gamma(\omega)$ and the characteristic impedance $Z_{c}(\omega)$ of the line are then given by (see e.g. [8]):

$$
\begin{aligned}
& \gamma(\omega)=\frac{1}{c} \sqrt{\omega_{c}^{2}-\omega^{2}} \\
& Z_{c}(\omega)=j Z_{0} \frac{\omega}{\sqrt{\omega_{c}^{2}-\omega^{2}}}
\end{aligned}
$$

where $c$ is the speed of light in free space, $\omega_{c}$ is the modal cutoff frequency and $Z_{0}$ is the free space intrinsic impedance. The source and the load are represented in the frequency domain by a voltage source $V_{S}(\omega)$ with a source impedance $Z_{S}(\omega)$ and by a load impedance $Z_{L}(\omega)$, respectively. Two cases which posses infinite multiple reflections will be considered here. Reflection at the load side is caused by a frequency independent resistive load $Z_{L}(\omega)=Z_{0}$ in both cases. Reflection at the generator side is caused by the zero source impedance of an ideal voltage source $\left(Z_{S}(\omega)=0\right)$ in the first case and by a frequency independent resistive source impedance $Z_{S}(\omega)=Z_{0}$ in the second case. In both cases a transfer function $H(\omega)$ is defined according to:

$H(\omega)=\frac{V_{L}(\omega)}{V_{S}(\omega)}$

where $V_{L}(\omega)$ is the load voltage in the frequency domain. The time dependent source and load voltages are obtained as inverse Fourier Transforms: 


$$
\begin{aligned}
& v_{S}(t)=\frac{1}{2 \pi} \int_{-\infty}^{+\infty} V_{S}(\omega) e^{j \omega t} d \omega \\
& v_{L}(t)=\frac{1}{2 \pi} \int_{-\infty}^{+\infty} V_{L}(\omega) e^{j \omega t} d \omega
\end{aligned}
$$

Let us now define the following spectral functions which will be used in the subsequent analysis:

$$
\begin{aligned}
& H_{0}(z, \omega)=e^{-\gamma(\omega) z} \\
& R_{n}(z, \omega)=\left[\frac{\sqrt{\omega_{c}^{2}-\omega^{2}}-j \omega}{\sqrt{\omega_{c}^{2}-\omega^{2}}+j \omega}\right]^{(n+1)} e^{-\gamma(\omega) z} \quad, n=0,1,2,3, \ldots
\end{aligned}
$$

The corresponding time dependent inverse Fourier transforms are given by:

$$
\begin{aligned}
& h_{0}(z, t)=\frac{1}{2 \pi} \int_{-\infty}^{\infty} e^{(j \omega t-\gamma(\omega) z)} d \omega \\
& r_{n}(z, t)=\frac{1}{2 \pi} \int_{-\infty}^{\infty}\left[\frac{\sqrt{\omega_{c}^{2}-\omega^{2}}-j \omega}{\sqrt{\omega_{c}^{2}-\omega^{2}}+j \omega}\right]^{(n+1)} e^{(j \omega t-\gamma(\omega) z)} d \omega \quad, n=0,1,2,3, \ldots
\end{aligned}
$$

It is worth noting that $R_{n}(z, \omega)$ and $r_{n}(z, t)$ posses the following properties:

$$
\begin{aligned}
& R_{n}\left(z_{1}, \omega\right) \cdot R_{m}\left(z_{2}, \omega\right)=R_{n+m+1}\left(z_{1}+z_{2}, \omega\right) \\
& r_{n}\left(z_{1}, t\right) * r_{m}\left(z_{2}, t\right)=r_{n+m+1}\left(z_{1}+z_{2}, t\right)
\end{aligned}
$$

where $*$ denotes the time-domain convolution. This applies to $H_{0}(z, \omega)$ and $h_{0}(z, t)$ as well if they were treated as being $R_{-1}(z, \omega)$ and $r_{-1}(z, t)$, respectively. As will be shown shortly, both $h_{0}(z, t)$ and $r_{n}(z, t)$ vanish identically for $t<(z / c)$.

\subsection{Case 1: $Z_{S}(\omega)=0, Z_{L}(\omega)=Z_{0}$}

Applying the classical transmission line theory (see e.g. [9]), it is readily proved that the corresponding transfer function $H_{l}(\omega)$ is given by:

$$
H_{1}(\omega)=\frac{H_{0}(L, \omega)+R_{0}(L, \omega)}{1+R_{0}(2 L, \omega)}
$$

In order to let $H_{l}(\omega)$ resemble the multiple reflection between the generator-side and the loadside of the waveguide section, (7-a) is rewritten as an infinite geometrical series and (6-a) is utilized:

$$
H_{1}(\omega)=\left[H_{0}(L, \omega)+R_{0}(L, \omega)\right] \cdot\left[1-R_{0}(2 L, \omega)+R_{1}(4 L, \omega)-R_{2}(6 L, \omega)+\cdots\right]
$$

The corresponding impulse response is then given by: 
$h_{1}(t)=\left[h_{0}(L, t)+r_{0}(L, t)\right] *\left[\delta(t)-r_{0}(2 L, t)+r_{1}(4 L, t)-r_{2}(6 L, t)+\cdots\right]$

where $\delta(t)$ denotes the Dirac-delta function. Making use of (6-b), the following simple expression for $h_{l}(t)$ is obtained:

$$
h_{1}(t)=h_{0}(L, t)+r_{0}(L, t)+\sum_{n=1}^{\infty}(-1)^{n}\left(r_{n-1}((2 n+1) L, t)+r_{n}((2 n+1) L, t)\right)
$$

The first load-side reflection is represented by the term $r_{0}(L, t)$ in the RHS of (7-d). The $n^{\text {th }}$ term of the infinite sum accounts for the $n+1^{\text {st }}$ load-side reflection, while its alternating sign accounts for the short-circuit reflection at the generator side due to the zero internal impedance of the ideal voltage source.

\subsection{Case 2: $Z_{S}(\omega)=Z_{0}, Z_{L}(\omega)=Z_{0}$}

The transfer function $H_{2}(\omega)$ representing this case is readily shown to be given by [9]:

$$
H_{2}(\omega)=\frac{H_{0}(L, \omega)-R_{1}(L, \omega)}{2\left[1-R_{1}(2 L, \omega)\right]}
$$

Following the same procedure as in the previous case, we arrive at:

$$
\begin{aligned}
& H_{2}(\omega)=\frac{H_{0}(L, \omega)-R_{1}(L, \omega)}{2} \cdot\left[1+R_{1}(2 L, \omega)+R_{3}(4 L, \omega)+R_{5}(6 L, \omega)+\cdots\right] \\
& h_{2}(t)=\frac{h_{0}(L, t)-r_{1}(L, t)}{2} *\left[\delta(t)+r_{1}(2 L, t)+r_{3}(4 L, t)+r_{5}(6 L, t)+\cdots\right]
\end{aligned}
$$

Each term in the above expansion represents in fact a pair of equal reflections (due to $Z_{S}=Z_{L}$ ); once at the generator side and once at the load side of the line section. Making use of (6-b), the following simple expression for $h_{2}(t)$ is obtained:

$$
h_{2}(t)=\frac{1}{2}\left(h_{0}(L, t)-r_{1}(L, t)+\sum_{n=1}^{\infty}\left(r_{2 n-1}((2 n+1) L, t)-r_{2 n+1}((2 n+1) L, t)\right)\right)
$$

\section{ANALYTIC EXPRESSIONS FOR $h_{0}(z, t)$ AND $r_{n}(z, t)$}

The key point in finding analytical expressions for $h_{0}(z, t)$ and $r_{n}(z, t)$ is a generic spectral function $G(z, \omega)$ whose inverse Fourier transform $g(z, t)$ is analytically integrable. These are given by:

$$
\begin{aligned}
& G(z, \omega)=\frac{e^{-\gamma(\omega) z}}{c \gamma(\omega)}=\frac{e^{-(z / c) \sqrt{\omega_{c}^{2}-\omega^{2}}}}{\sqrt{\omega_{c}^{2}-\omega^{2}}} \\
& g(z, t)=\frac{1}{2 \pi} \int_{-\infty}^{\infty} \frac{e^{\left(j \omega t-(z / c) \sqrt{\omega_{c}^{2}-\omega^{2}}\right)}}{\sqrt{\omega_{c}^{2}-\omega^{2}}} d \omega
\end{aligned}
$$

The integral in (9-b) has the following closed-form expression [10]: 
$g(z, t)=u\left(t-\frac{z}{c}\right) J_{0}\left(\omega_{c} \sqrt{t^{2}-\left(\frac{z}{c}\right)^{2}}\right)$

where $u(x)$ and $J_{n}(x)$ are the unit step function and the $n^{\text {th }}$ order ordinary Bessel function (here $n=0)$, respectively. Expressing all already defined spectral functions as

$F(z, \omega)=\tilde{F}(\gamma, \omega) e^{-\gamma z}$ and dealing with $\omega$ and $j \gamma$ as being the temporal and spatial frequency corresponding to $t$ and $z$, respectively, results in the following simple rules:

$$
\frac{\partial f}{\partial t} \leftrightarrow j \omega \tilde{F} \quad \frac{\partial f}{\partial z} \leftrightarrow-\gamma \tilde{F}
$$

where $f(z, t)$ is the inverse Fourier transform of $F(z, \omega)$. The frequency dependence of $\gamma$ given in (1-a) results then in:

$c^{2} \frac{\partial^{2} f}{\partial z^{2}}-\frac{\partial^{2} f}{\partial t^{2}}=\omega_{c}^{2} f$

Making use of (11), the following recursive relations are easily obtained:

$$
\begin{aligned}
& h_{0}(z, t)=-c \frac{\partial}{\partial z} g(z, t) \\
& r_{0}(z, t)=\frac{2}{\omega_{c}^{2}} \frac{\partial}{\partial t}\left(\frac{\partial h_{0}}{\partial t}+c \frac{\partial h_{0}}{\partial z}\right)+h_{0}(z, t)=\frac{2 c}{\omega_{c}^{2}} \frac{\partial}{\partial z}\left(\frac{\partial h_{0}}{\partial t}+c \frac{\partial h_{0}}{\partial z}\right)-h_{0}(z, t) \\
& r_{n+1}(z, t)=\frac{2}{\omega_{c}^{2}} \frac{\partial}{\partial t}\left(\frac{\partial r_{n}}{\partial t}+c \frac{\partial r_{n}}{\partial z}\right)+r_{n}(z, t)=\frac{2 c}{\omega_{c}^{2}} \frac{\partial}{\partial z}\left(\frac{\partial r_{n}}{\partial t}+c \frac{\partial r_{n}}{\partial z}\right)-r_{n}(z, t)
\end{aligned}
$$

Combining (10) and (12-a) results in the analytical expression for $h_{0}(z, t)$ :

$h_{0}(z, t)=\delta\left(t-\frac{z}{c}\right)-u\left(t-\frac{z}{c}\right) \frac{\omega_{c}\left(\frac{z}{c}\right) J_{1}\left(\omega_{c} \sqrt{t^{2}-\left(\frac{z}{c}\right)^{2}}\right)}{\sqrt{t^{2}-\left(\frac{z}{c}\right)^{2}}}$

In order to find explicit expressions for $r_{n}(z, t)$ we introduce the following variables:

$$
\theta=\omega_{c}\left(t+\frac{z}{c}\right) \quad \phi=\omega_{c}\left(t-\frac{z}{c}\right) \quad \lambda=\sqrt{\theta \phi}
$$

It is easily shown that all $r_{n}(z, t)$ don't include $\phi$-derivatives and the recurrence relations (12-b) and (12-c) take then the following simple forms:

$$
\begin{aligned}
& r_{0}(z, t)=4 \frac{\partial^{2} h_{0}}{\partial \theta^{2}}=\left(\frac{\phi}{\lambda}\right)^{2}\left(\frac{\partial^{2} h_{0}}{\partial \lambda^{2}}-\frac{1}{\lambda} \frac{\partial h_{0}}{\partial \lambda}\right) \\
& r_{n+1}(z, t)=4 \frac{\partial^{2} r_{n}}{\partial \theta^{2}}=\left(\frac{\phi}{\lambda}\right)^{2}\left(\frac{\partial^{2} r_{n}}{\partial \lambda^{2}}-\frac{1}{\lambda} \frac{\partial r_{n}}{\partial \lambda}\right)
\end{aligned}
$$


Using induction, the following analytical expression for $r_{n}(z, t)$ is obtained:

$$
r_{n}(z, t)=\frac{\omega_{c}}{2} u\left(\frac{\phi}{\omega_{c}}\right)\left(\left(\frac{\phi}{\lambda}\right)^{2 n+3} J_{2 n+3}(\lambda)+\left(\frac{\phi}{\lambda}\right)^{2 n+1} J_{2 n+1}(\lambda)\right), \quad n=0,1,2,3, \ldots
$$

\section{NUMERICAL RESULTS}

Due to the space limitations, we will restrict the numerical results to case 2 only. For the graphical demonstration of the dispersion related to the different terms in (8-d), the source voltage $v_{S}(t)$ has been assumed to be a narrowband Gaussian base-band pulse modulating a carrier signal, which is switched on at $t=0$ :

$v_{S}(t)=V_{0} u(t) e^{-\left(\frac{t-t_{m}}{\tau}\right)^{2}} \cos \omega_{\text {carrier }}\left(t-t_{m}\right)$

where $V_{0}$ is the pulse maximum which is reached at $t=t_{m}, \tau$ is the half pulse width and $\omega_{\text {carrier }}$ is the carrier frequency. The corresponding spectrum $V_{S}(\omega)$ is a Gaussian pulse of a half pulse width $(2 / \tau)$, which is centered at $\omega_{\text {carrier }}$ (see e.g. [10]). The load voltage $v_{L}(t)$ is obtained as a convolution of $v_{S}(t)$ in (17) with $h_{2}(t)$ in (8-d), which results in:

$v_{L}(t)=v_{L 0}(t)+v_{L 1}(t)+\sum_{i=2}^{\infty} v_{L i}(t)$

where the different terms in (18) are the convolution of $v_{S}(t)$ with the corresponding terms in (8-d). It is readily seen that $v_{L 0}(t)$ represents the forward transmission, while $v_{L i}(t)$ $(i=1,2,3, \ldots)$ represents the $i^{\text {th }}$ load-side reflection. The latter are echoes for $i \geq 2$. The delayed source voltage $v_{S}\left(t-\frac{L}{c}\right)$ is a part of $v_{L 0}(t)$, as $h_{0}(L, t)$ contains $\delta\left(t-\frac{L}{c}\right)$ (refer to (13)).

In order to deal with normalized quantities which can be scaled to any frequency band and any waveguide dimensions, a normalizing frequency $\omega_{0}$ is introduced:

$$
\begin{aligned}
& v_{S}(\theta)=V_{0} u(\theta) e^{-\left(\frac{\theta-\theta_{m}}{\theta_{\tau}}\right)^{2}} \cos \varpi_{\text {carrier }}\left(\theta-\theta_{m}\right) \\
& v_{L}(\theta)=v_{L 0}(\theta)+v_{L 1}(\theta)+\sum_{i=2}^{\infty} v_{L i}(\theta)
\end{aligned}
$$

where $\theta=\omega_{0} t, \theta_{m}=\omega_{0} t_{m}, \theta_{\tau}=\omega_{0} \tau, \varpi_{\text {carrier }}=\omega_{\text {carrier }} / \omega_{0}$ and $\theta_{0}=\omega_{0} L / c$. A value of $\varpi_{\text {carrier }}=5$ will be used in all cases considered below. In order to have a dominating steady-state response, $\theta_{m}=5 \theta_{\tau}$ will be used, which leads to greatly depressing the excitation of the transient response accompanying the switch-on process at $\theta=0$. 
We first consider a case with $\theta_{\tau}=\pi, \theta_{0}=5 \pi$ and $\varpi_{c}=\omega_{c} / \omega_{0}=4$. This corresponds to a relative bandwidth of $V_{S}(\omega)$ of about $25 \%$. Most of this spectrum lies above the cutoff frequency $\omega_{c}$. Fig. 2 shows the first few terms $v_{L i}(\theta)$. In Fig. 2-a, $v_{S}\left(\theta-\theta_{0}\right)$, which represents the undistorted output, is plotted for the sake of comparison. Figs. 2-b and 2-c show the forwardtransmission term $v_{L 0}(\theta)$ and the first load-side-reflection term $v_{L 1}(\theta)$, respectively. Figs. 2$\mathrm{d}$ and 2-e show, on the other hand, the first two echoes $v_{L 2}(\theta)$ and $v_{L 3}(\theta)$, respectively. The dispersion in $v_{L 0}(\theta)$ is mainly due to the spectral components of $V_{S}(\omega)$ in the vicinity of the cutoff frequency $\omega_{c}$. The corresponding group velocity is very small, which results in a very long transit time for these spectral components. This causes the long tail at the trailing edge of $v_{L 0}(\theta)$. It is worth noting that late echoes are more dispersed than earlier ones. This is due to the fact that the propagation length of late echoes is longer than that of earlier ones.

In order to justify the long-tail explanation given above, we let the carrier frequency of the source voltage coincide with the cutoff one $\left(\varpi_{\text {carrier }}=\varpi_{c}=5\right)$, so that most of the spectrum $V_{S}(\omega)$ lies around $\omega_{c}$. All other parameters remain unchanged. Fig. 3 shows the corresponding $v_{L 0}(\theta)$, where the major contribution is now due to the upper half of $V_{S}(\omega)$, which lies above cutoff. The group velocity corresponding to the spectral components belonging to this half spectrum covers the range from zero (at $\omega=\omega_{c}$ ) to a fraction of $c$. This results in a very wide range for the transit time of the different spectral components. The latter leads to the very long tail at the trailing edge of $v_{L 0}(\theta)$ in Fig. 3.

We consider next a case where most of the source-voltage spectrum lies below cutoff. The values $\varpi_{c}=5.5$ and $\theta_{\tau}=5 \pi$ (corresponding to a relative bandwidth of $V_{S}(\omega)$ of about $5 \%$ ) fulfill this requirement. Figs. 4-a and 4-b show $v_{S}\left(\theta-\theta_{0}\right)$ and $v_{L 0}(\theta)$, respectively, for a relatively short waveguide section $\left(\theta_{0}=2 \pi\right)$ (all other parameters remain unchanged). It is readily seen that the propagation of the pulse peak is superluminal in this case (as the pulse peak of $v_{L 0}(\theta)$ appears earlier than that of $\left.v_{S}\left(\theta-\theta_{0}\right)\right)$. Another interesting feature in this case is the slight temporal pulse compression accompanying the transmission, which is easily observed by comparing the pulse width in Figs. 4-a and 4-b. This phenomenon has been investigated in details in our recent publication [7].

The above observed superluminal propagation is in fact very tricky. It appears in electrically short waveguide sections only. It is destroyed if the waveguide section becomes electrically long. The reason is the temporal pulse compression accompanying the transmission, which increases as the waveguide section becomes longer. This results in a proportional spectral pulse broadening which shifts a part of the signal spectrum towards the cutoff frequency. As the length of the waveguide section increases, the higher spectral edge of the signal spectrum becomes above cutoff. Due to the high pass effect of the below-cutoff transmission, this spectral edge becomes dominating, and the tail effect mentioned above takes place, which destroys the above observed superluminality.

In order to justify the above discussion, we increased the waveguide length of the last case from $\theta_{0}=2 \pi$ to $\theta_{0}=5 \pi$. Figs. 5-a and 5-b show the corresponding $v_{S}\left(\theta-\theta_{0}\right)$ and $v_{L 0}(\theta)$, respectively. No superluminality can be observed in this case, which justifies the explanation given above. 


\section{CONCLUSION}

Transfer functions and their corresponding impulse responses, which are related to waveguide signal transmission have been analytically derived. Dispersion of the forward transmission and the multiple reflections (echoes) of a carrier signal modulated by a narrowband Gaussian base-band pulse has been graphically demonstrated. Recently reported superluminal propagation of pulse peaks in evanescent waveguides has been shown to take place in electrically short waveguide sections only. Increasing the waveguide length beyond a certain limit destroys such a superluminality.

\section{REFERENCES}

[1] CARLSON, A.B.: 'Communication Systems' (McGraw-Hill, 1986)

[2] BRILLOUIN, L.: 'Wave Propagation and Group Velocity’ (Academic Press, 1960)

[3] JACKSON, J.D.: 'Classical Electromagnetics' (Wiley, 1975)

[4] KARBOWIAK, E.: 'Propagation of transients in waveguides', Proc. IEE (C), vol. 104, 1957, pp. 339-349

[5] DVORAK, S.L.: 'Exact, closed-form expressions for transient fields in homogeneously filled waveguides', IEEE Trans. Microwave Theory Tech., vol. 42, 1994, pp. 2164-2170

[6] OMAR, A.S. and KAMEL, A.H.: 'Network theoretical transient analysis of signal transmission over evanescent modes', will appear in IEEE Trans. Antennas Propagat., March 2003

[7] OMAR, A.S. and KAMEL, A.H.: 'Steady state analysis of signal transmission in evanescent channels', will appear in IEEE Trans. Antennas Propagat., May 2003

[8] COLLIN, R.E.: 'Field Theory of Guided Waves' (IEEE Press, 1991)

[9] COLLIN, R.E.: 'Foundations for Microwave Engineering' (McGraw-Hill, 1966)

[10] ABRAMOWITZ, M. and STEGUN, I.A.: 'Handbook of Mathematical Functions' (Dover Publications, 1972) 


\section{Figure Captions}

Fig. 1: $\quad$ Equivalent line section with source and load terminations.

Fig. 2: $\quad$ Delayed source voltage $v_{S}\left(\theta-\theta_{0}\right)(2-\mathrm{a})$, forward-transmission term $v_{L 0}(\theta)$ (2b), first load-side-reflection term $v_{L 1}(\theta)(2-c)$, first echo $v_{L 2}(\theta)(2-\mathrm{d})$ and second echo $v_{L 3}(\theta)$ (2-e) for the case with:

$\varpi_{\text {carrier }}=5, \varpi_{c}=4, \theta_{m}=5 \pi, \theta_{\tau}=\pi$ and $\theta_{0}=5 \pi$.

Fig. 3: Forward-transmission term $v_{L 0}(\theta)$ for the case with:

$\varpi_{\text {carrier }}=5, \varpi_{c}=5, \theta_{m}=5 \pi, \theta_{\tau}=\pi$ and $\theta_{0}=5 \pi$.

Fig. 4: Delayed source voltage $v_{S}\left(\theta-\theta_{0}\right)$ (4-a) and forward-transmission term $v_{L 0}(\theta)$ (4-b) for the case with:

$\varpi_{\text {carrier }}=5, \varpi_{c}=5.5, \theta_{m}=25 \pi, \theta_{\tau}=5 \pi$ and $\theta_{0}=2 \pi$.

Fig. 5: Delayed source voltage $v_{S}\left(\theta-\theta_{0}\right)(5-a)$ and forward-transmission term $v_{L 0}(\theta)$ (5-b) for the case with:

$\varpi_{\text {carrier }}=5, \varpi_{c}=5.5, \theta_{m}=25 \pi, \theta_{\tau}=5 \pi$ and $\theta_{0}=5 \pi$. 


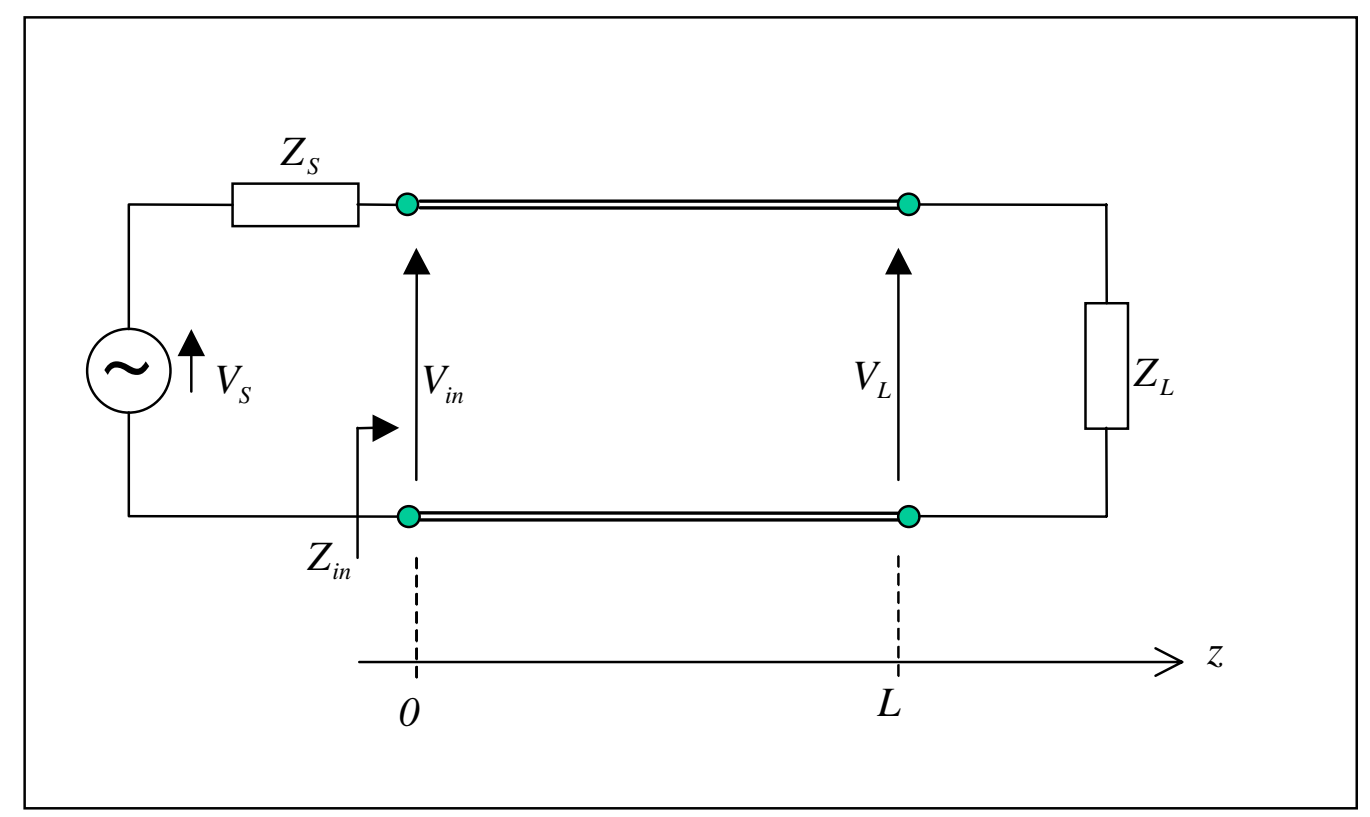

Fig. 1 


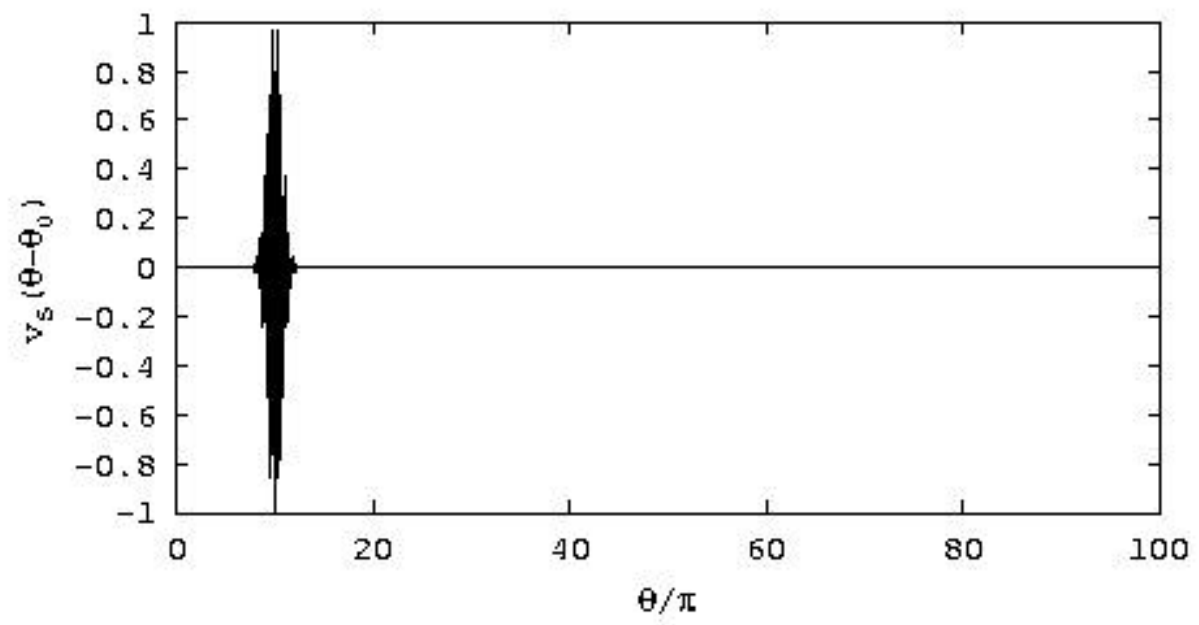

Fig. 2-a

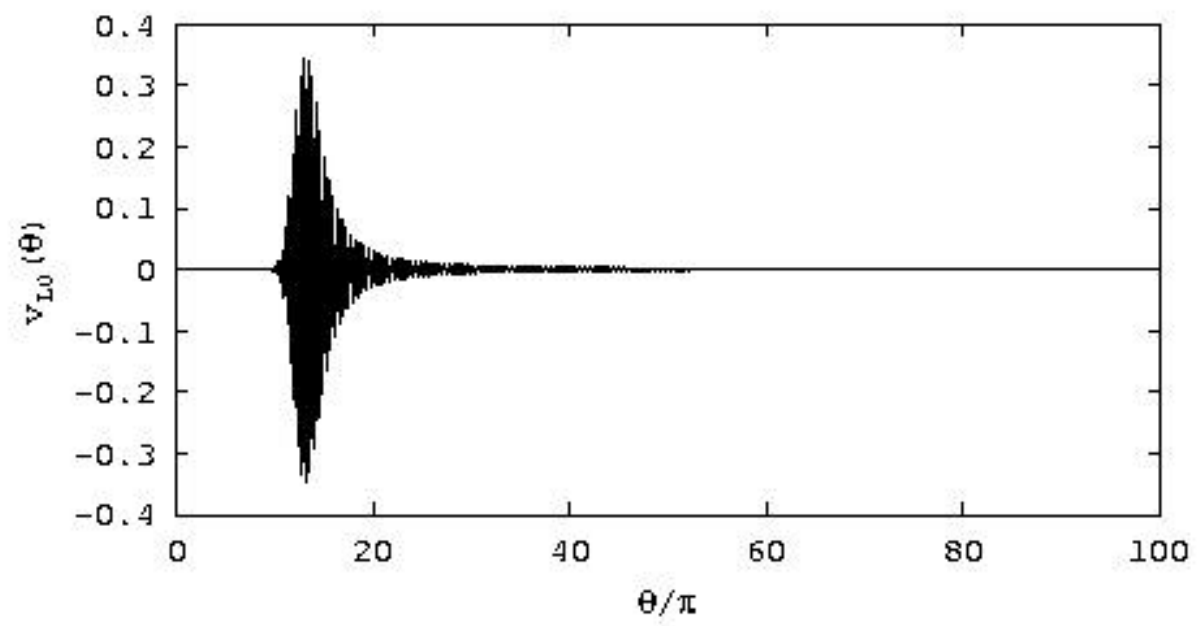

Fig. 2-b

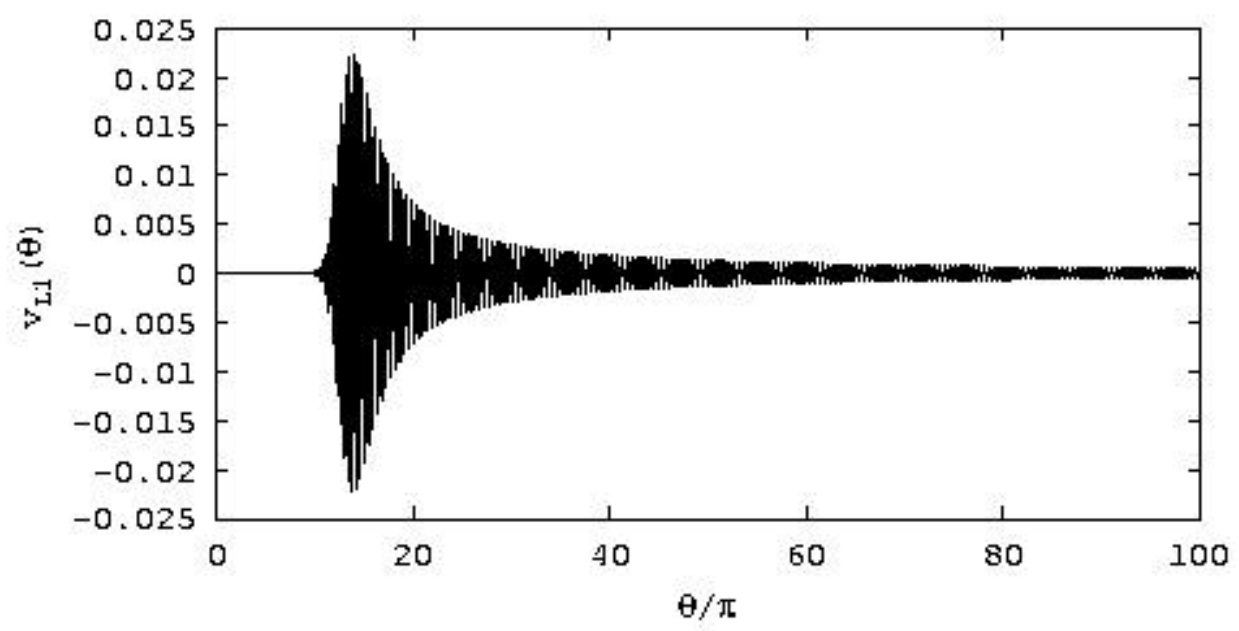

Fig. 2-c 


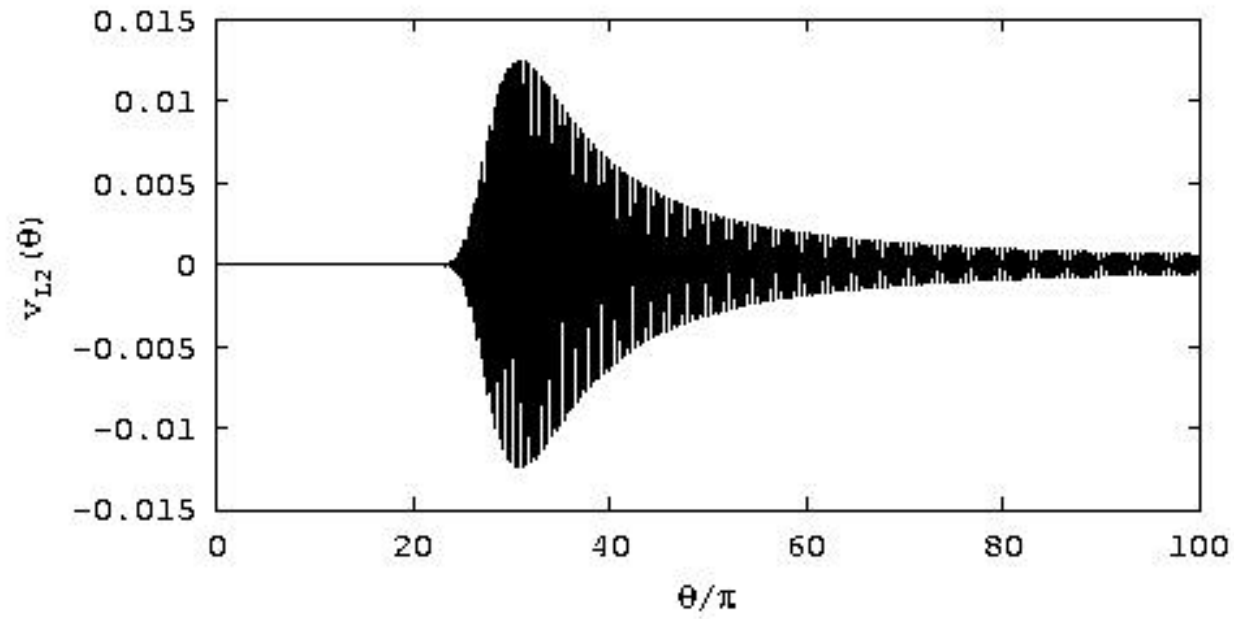

Fig. 2-d

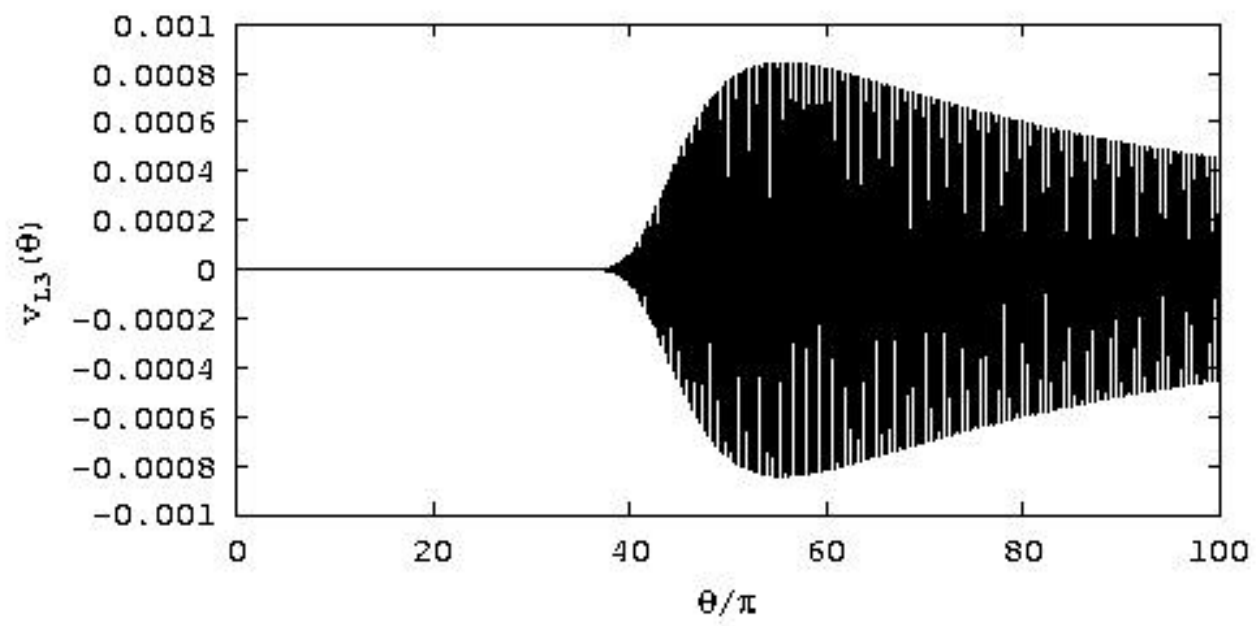

Fig. 2-e

Fig. 2 


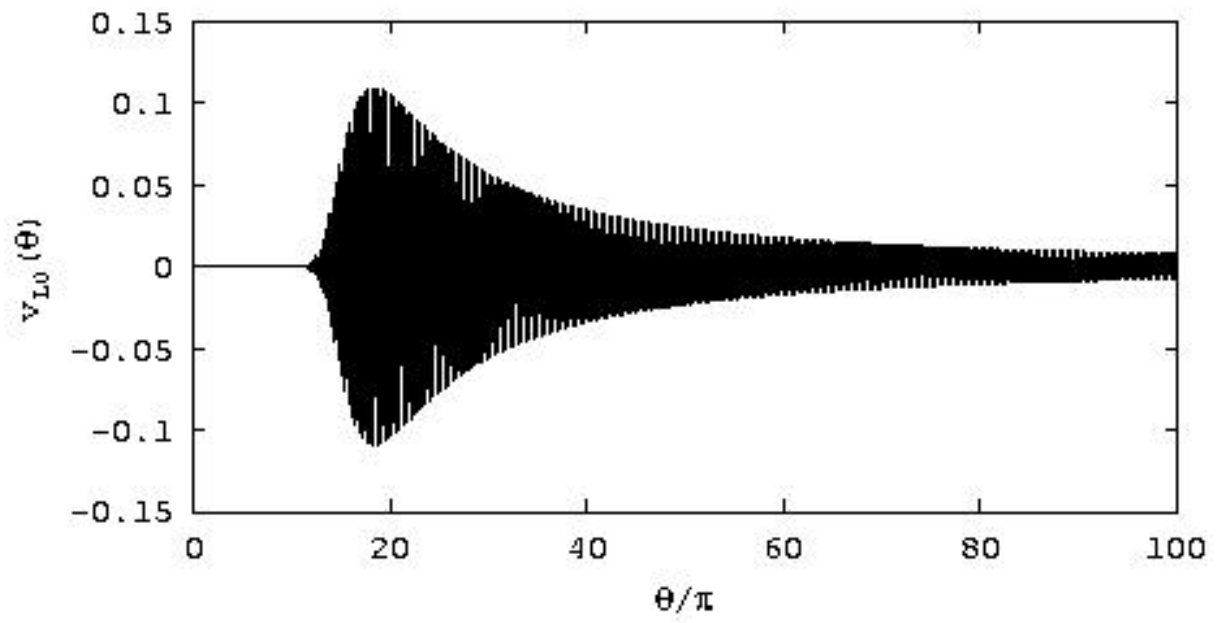

Fig. 3 


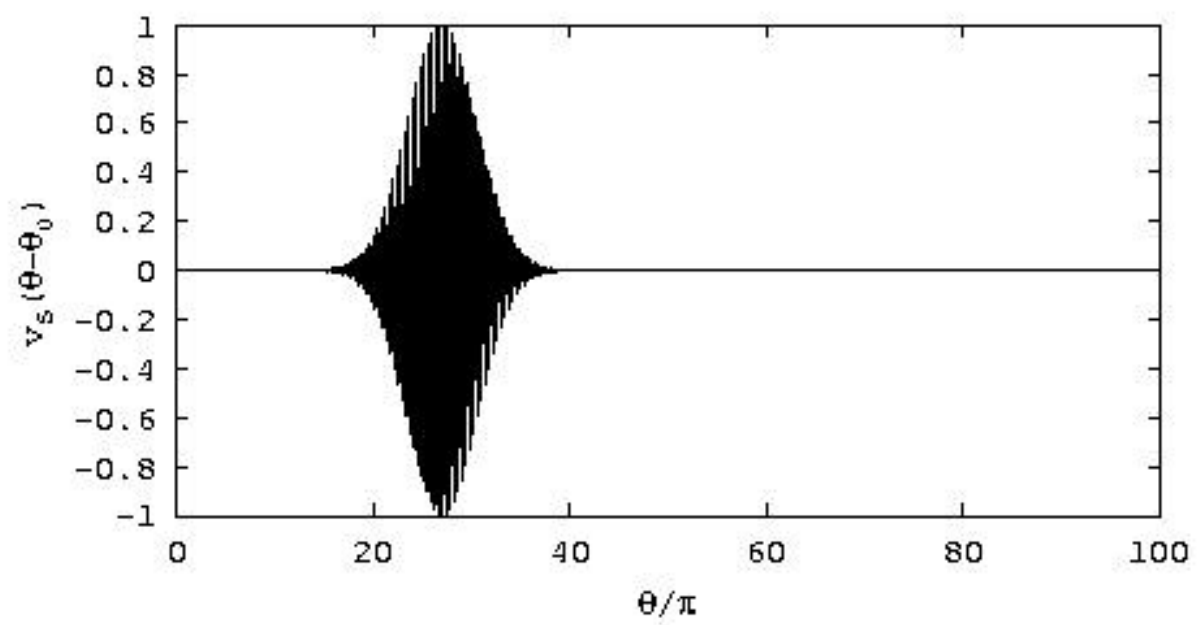

Fig. 4-a

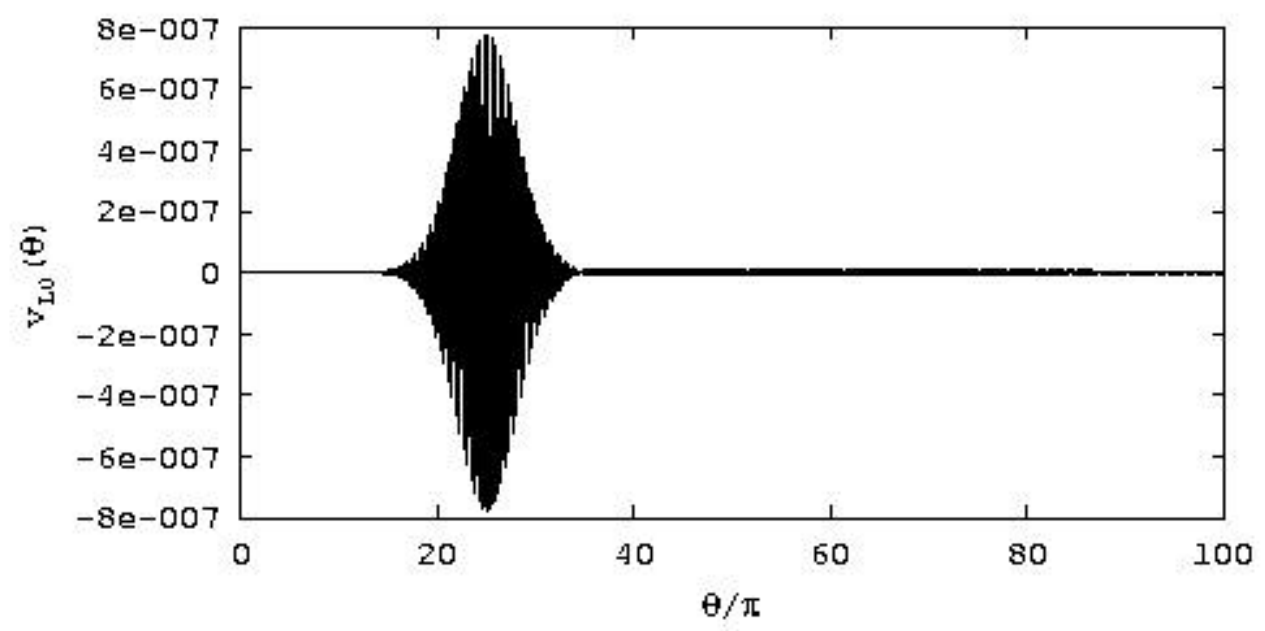

Fig. 4-b

Fig. 4 


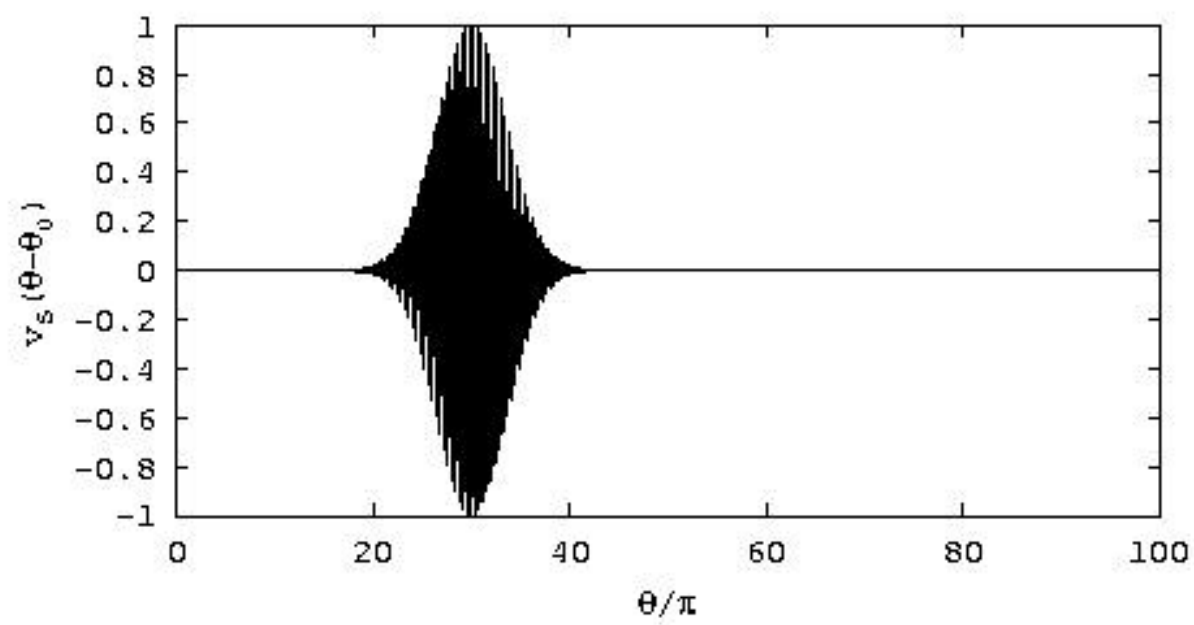

Fig. 5-a

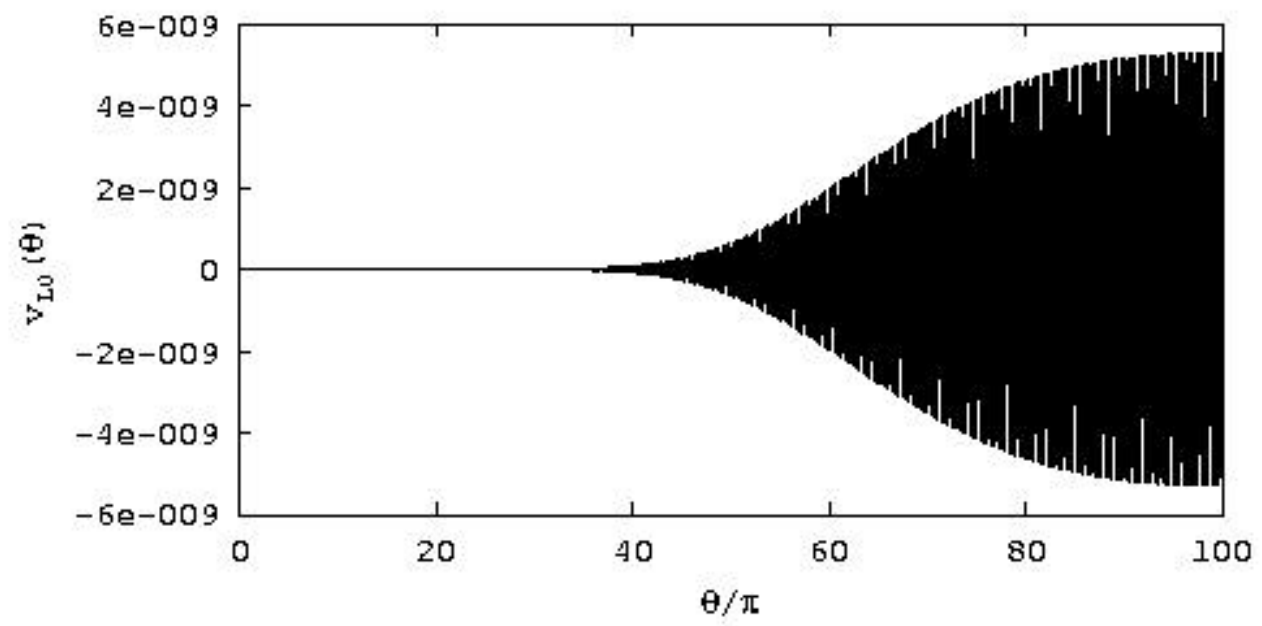

Fig. 5-b

Fig. 5 